\title{
NETWORKS OF NEURONS AS DYNAMICAL SYSTEMS: FROM GEOMETRY TO BIOPHYSICS
}

\author{
BY \\ NANCY KOPELL \\ Department of Mathematics, Center for Biodynamics, Boston University
}

1. Introduction. This talk is about an area I believe will present many mathematical challenges in the near future: the border between the nervous system and dynamical systems. The central nervous system is almost overwhelmingly complex. One task of the people who study it is to try to connect the "wetware" (neurons and networks of them) with behavior such as seeing, moving, and proving theorems. That is, the problem is to "reverse-engineer" the nervous system to figure out what it is doing and how it is doing it. This is an enormous task shared by tens of thousands of neuroscientists. I am going to discuss a possible role for dynamical systems in this very large enterprise: as a tool for teasing out which details are important for which behavior.

Though dynamical systems ideas are even more broadly useful, this talk focuses on rhythmic behavior in networks of neurons. Rhythms with a large range of frequencies are found all over the nervous system, correlated with motor activities [1], sensory processing (visual [2] and olfactory [3]), attention [4], learning [5], and pathological states such as Parkinson's tremor [6] and epilepsy [7]. What is not at all yet understood is how networks are constructed to carry out their tasks, and what role dynamics plays in that. I shall discuss several case studies, to show how dynamical systems methods can unearth nonintuitive modes of operation. Each of the case studies raises some issue about synchronization of rhythmic systems.

The work to be described here is in contrast to some earlier work with Bard Ermentrout [8], [9] concerning a motor network regulating undulatory locomotion (swimming) in fish and fish-like animals. The mathematical punchline of that work was that some kinds of dynamical behavior are so robust and so general that focusing on cellular details only obscures the explanations for the behavior. In the case of undulatory locomotion, the relevant network is, roughly, an array of oscillators arranged in a chain; the mathematics was used to show that this structure alone could account for much of the observed behavior.

The work described here can be seen as a counterpoint to that work, and shows another way in which dynamical systems can be useful. Here the emphasis is on the detailed

Received November 19, 1997.

1991 Mathematics Subject Classification. Primary 34C, 92C20.

(C)1998 Brown University 
dynamics of cells and connections between them, which can have major implications for how networks of neurons can operate. The case studies illustrate how biological structure may translate into mathematical structure, which can then be exploited to help connect properties from cellular biophysics with network behavior.

2. Some biological and mathematical background, and a mystery. Physically, neurons are of many sizes and shapes [10]. Though this is thought to be important in how neurons interact with other neurons to produce network behavior, it is not the subject of this talk (though some of the case studies have implications for that). Instead, I shall be focusing on a complementary kind of complexity: the biophysics of patches of membrane. The mathematical description of this goes back to the Nobel Prize-winning work of Hodgkin and Huxley in the early 1950s [10].

Electrical activity of an individual neuron is associated with changes in cross-membrane potential (voltage) due to flows of charged ions across the membrane. The ions flow through channels that open and close in ways that are "gated" (i.e., controlled) by the voltage of the cell. In its simplest (and inaccurate) form, the voltage of the cell is considered to be the same at all points of the cell, and the equation for the change in voltage has the form [11]

$$
C d v / d t=-\sum I_{\text {ion }}-I_{\text {appl }} .
$$

Here $v$ is the voltage (cross-membrane potential) of the cell, and $C$ is the capacitance. $I_{\text {ion }}$ represents one of the ionic flows across the membrane. $I_{\text {appl }}$ is the applied current imposed by the experimenter. There are also equations for each of the ionic flows, which describe the process of opening and closing channels. The channel openings are stochastic [12], but if there are enough channels, there is a deterministic limit. A given ionic flow may have more than one gate (known as "activation" and "inactivation"), both of which must be open for current to flow. An ionic current is usually described by the form

$$
I=\bar{g} m^{k} h^{j}\left(v-v_{R}\right)
$$

where $\bar{g}$ is the maximal conductance, $m$ and $h$ represent the fraction of each type of gate that is open and $v_{R}$ is the "reversal potential" of the specific current; at that potential, the current changes between inward (depolarizing, or pushing the cell toward firing) and outward (hyperpolarizing, or pushing the cell away from firing). The quantities $m$ and $h$ satisfy equations of the form

$$
x^{\prime}=\left(x_{\infty}(v)-x\right) / \tau_{x},
$$

where $m_{\infty}(v)$ increases with $v$ and $h_{\infty}(v)$ decreases with $v$. There is also a "leak" current, given by $\bar{g}_{\mathrm{L}}\left(v-v_{\mathrm{L}}\right)$, with no gates. In the fairly simple circuit described by Hodgkin and Huxley, a short depolarizing change in voltage past some threshold opens the sodium $\left(\mathrm{Na}^{+}\right)$activation gate, leading to an inward flow of $\mathrm{Na}^{+}$ions that makes the voltage undergo further change in the same direction (less negative, and then positive). The inactivation of the sodium, and the activation of the outward flowing potassium $\left(\mathrm{K}^{+}\right)$ turn on with a longer time constant at the higher voltage level produced by the sodium flow; this reverses the change in the membrane potential. These processes together produce the classic "action potential" (or "spike") once the cell is depolarized past the 
threshold. With sufficient applied current or more (and more complicated) ionic currents, some cells can produce "bursts" of spikes interspersed with silent periods. (See [11] for a mathematical treatment.)

Time constants can be very different from channel to channel and gate to gate, and generally much larger than the voltage equations; so this is a system with many different time scales. The above complication is only the beginning. For example, channels can be gated not just by voltage but also by other quantities, notably the concentration of calcium [13]. Different cells have different combinations of channels, with different conductances. Even a single cell may express a different assortment of channels in its soma than in its dendrites, and the voltages at different parts of the cell are related by (possibly nonlinear) versions of the cable equation [14], rather than being equal. Furthermore, there are many "neuromodulators" that act on all of the quantities in these equations, with changes that are themselves on different time scales [15], [16], and which themselves can depend on the previous activity of the network [17]. Thus, the problem of reverse engineering includes the mystery of why the nervous system is constructed to be so complicated.

3. Some simpler mathematical descriptions. It is almost impossible to attack this complexity in a frontal manner. The strategy is rather to sneak up on the mystery, using reductions of the complex descriptions that capture some (but only some) of the basic behavior of the neurons. The simplest models of this type are two-dimensional, with several ionic currents but only one current having a gate with dynamics. The much used Morris-Lecar model [18], [11], used to model a broad calcium-based spike, or (phenomenologically) to describe the voltage envelope of a neuron with periodic bursts of spikes, has the form

$$
\begin{aligned}
v^{\prime} & =-\bar{g}_{\mathrm{Na}} m_{\infty}(v)\left(v-v_{\mathrm{Na}}\right)-\bar{g}_{\mathrm{K}} w\left(v-v_{\mathrm{K}}\right)-\bar{g}_{\mathrm{L}}\left(v-v_{\mathrm{L}}\right), \\
w^{\prime} & =\left(w_{\infty}(v)-w\right) / \tau_{w} .
\end{aligned}
$$

There is a calcium current, a potassium current, and a leak current. For the calcium current, the activation is sufficiently fast that $m$ can be treated as an instantaneous function of $v$, rather than satisfying a differential equation. The potassium current has an activation, but no inactivation, leading to just one auxiliary equation.

This is a "singularly perturbed" or "fast-slow" system if $1 / \tau_{w} \ll 1$. In some parameter ranges, (1) has the same phase-plane behavior as a van der Pol oscillator and similar nullclines. The nullcline $v^{\prime}=0$ is qualitatively cubic shaped, and $w^{\prime}=0$ is the graph of $w=w_{\infty}(v)$, an increasing sigmoid that saturates for $v$ small and $v$ large. The oscillating regime occurs for parameters such that $w=w_{\infty}(v)$ intersects $v^{\prime}=0$ on its middle branch. In other regimes, it can have stable rest points at both high and low voltages, or be excitable (a sufficient perturbation from a stable critical point leads to a burst, and then returns to rest). By modifying the time scales, it is also possible to get twodimensional models whose voltage activity is spiking, rather than bursting [19]. Such 2-D models do not capture the dynamics of more complicated ionic flows, but they are adequate for the case studies in hand, which deal more with the properties of coupling than with those of the cells themselves. 
Cells interact with each other in many ways, most notably through chemical and electrical synapses that connect pairs of neurons. Like the cells, the synapses are biologically very complicated [10], and the models used are phenomenological reductions of very complex sets of processes.

A classic fast chemical synapse is often modeled as providing an additional "synaptic" current to the right-hand side of (1), with the form $\bar{g} m_{\infty}(v)\left(v-v_{\text {syn }}\right)$, where $v$ is the voltage of the "post-synaptic" cell receiving the current, and $\bar{v}$ is the voltage of the "pre-synaptic" cell that gates this current. The reversal potential $v_{\text {syn }}$ of the synapse determines whether the current is inhibitory (outward) or excitatory (inward). If $m_{\infty}(\bar{v})$ is, as often used, a saturating sigmoidal function, then the current can be considered zero for sufficiently low $\bar{v}$, and at its maximum $\bar{g}\left(v_{\text {syn }}-v\right)$ for $\bar{v}$ large enough. A mathematical effect of adding such a current, for $\bar{v}$ large enough, is to change the nullsurface $v^{\prime}=0$, raising it (with a change in shape) for an excitatory synapse, and lowering it (with a change in shape) for an inhibitory one. This effectively changes the threshold for spiking or bursting, leading to the terminology "fast threshold modulation" [22] for this kind of coupling. For synapses that have finite rise times or decay times, further equations must be added for the gates, as for the intrinsic cellular currents.

Electrical coupling has a simpler mathematical description, but turns out to lead to harder mathematical questions. An unrectifying electrical synapse adds current to each cell proportional to the difference $\pm|d(v-\bar{v})|$ between its voltage and that of its partner. Thus, it is mathematically the same as diffusion across a membrane between discrete containers.

4. Fast synapses and the effect of oscillator waveform. All of the case studies below focus on issues related to what makes a network synchronize, and they are all very different. As discussed in the introduction, the subtext of the talk is that biophysical structure leads to mathematical structure in the description of the cells or coupling, and that mathematical structure can have consequences at the network level. Sometimes it is only a very small amount of that structure that matters, and that is the punchline of the first case study.

Because of the different time scales between the voltage and other equations, individual cells in a network that are oscillators (bursters) are likely to have the wave form of relaxation oscillation. That is, the wave form of the envelope of spikes of neurons has plateaus at high and low voltage, with rapid switches in between. The wave form (independent of what currents produce it) turns out to have implications when such bursters are coupled by fast chemical synapses.

To make this point, I must first digress to talk about non-relaxation oscillators, with no multiple time scales in the wave form. With arbitrary limit cycle equations, and "weak enough", but otherwise arbitrary, coupling, such coupled oscillators can be reduced with no further loss of generality - to phase oscillators coupled through the differences of their phases. That is, they behave like equations of the form

$$
\theta_{j}^{\prime}=\omega_{j}+\sum_{k} H\left(\theta_{k}-\theta_{j}\right)
$$


where $\omega_{j}$ is the natural frequency of the $j$ th uncoupled oscillator, and $H\left(\theta_{k}-\theta_{j}\right)$ encodes the changes to that frequency due to the $k$ th oscillator.

The reduction to Eq. (2), which uses invariant manifold theory and averaging [20], is very general; all it takes is weak enough coupling, and sometimes it can be shown to be valid even for strong coupling [21]. However, it can fail for some kinds of coupling, and does so for the kind of "fast threshold modulation" coupling I described as the action of a fast chemical synapse. To get reduction to a phase model for this coupling, the coupling must be extremely weak; otherwise, the mathematical structure of the coupling is very different. The difference in structure shows up in the difference in behavior of the arrays of oscillators coupled by fast synapses, versus phase models. Consider, for example, chains of oscillators coupled locally (to their nearest neighbors). It follows from the analysis done with Bard Ermentrout (motivated by undulatory swimming) that, generically, any coupling mechanism that can be reduced to equations describing interactions of phases produces traveling waves of activity [8]. This comes essentially from edge effects of the chain (less coupling input to the outer two oscillators of a finite chain), which propagate through the chain no matter how long it is. Chains of relaxation oscillators with FTM coupling behave very differently [22], [23]. David Somers and I showed that edge effects in those equations can be kept local, with the oscillators responding to edge effects by locally changing wave amplitude instead of producing phase differences. Thus, stable synchronization of jumps between plateaus is possible (in the limit $\varepsilon \rightarrow 0$ ).

The two different kinds of oscillators also display differences in speeds of transients to locking. For the phase oscillators, the transients are very slow to decay. The relaxation oscillators synchronize very fast [22], [24]. Fast synchronization is necessary for some conjectured uses of oscillations in the nervous system. One such is a potential contribution to understanding the "binding problem" of how assemblies of cells, possibly in different parts of the nervous system, can act together to produce, e.g., a motion or a percept [25]. The ideas have also been used to explain aspects of image segmentation in the visual system [26].

5. Electrical coupling. This story and the next one focus on the structure of the coupling between cells and some unintuitive consequences. This section concerns electrical coupling, which is modeled (for nonrectifying gap junctions) as discrete diffusion. Since diffusion can be effective at homogenizing temperatures or concentrations, it is not surprising that it is effective at synchronizing cells that are dynamically similar. However, electrical synapses often connect cells that have very different dynamical properties. For example, one cell may be an oscillator while the other is bistable or excitable. The question then is: what is the electrical coupling doing?

The particular example I am focusing on here comes from a small, very well-studied invertebrate network in lobsters and crabs, called the "stomato-gastric ganglion" or STG [27]. The network controls the stomach, where the animal chews its food. It has a few dozen cells, individually recognizable, and a complicated pattern of connections among the cells. In spite of much knowledge about the biophysical properties of the individual cells, it remains to be understood how the cells all work together to produce the functionally important behavior. 
An important subset of the STG is a pair of dynamically different cells that form the "pacemaker" of one of the subnetworks of the STG network. The cell known as the "AB" cell can spontaneously oscillate. It is strongly coupled electrically to a pair of cells known as the "PD" cells, which can be bistable at high and low voltage when uncoupled. The $\mathrm{AB} / \mathrm{PD}$ subset has some strikingly different behavior from the isolated $\mathrm{AB}$ cell: When current is fed into the $\mathrm{AB}$ cell, the frequency changes by changing only the part of the cycle in which the cell is inactive (not spiking). But when current is fed into an AB cell electrically coupled to the PD cells, both the active (spiking) part of the cycle and the inactive part change, and in such a way as to keep their ratio constant. In a simulation [28], Abbott, Marder, and Hooper suggested a way in which the constant ratio could be achieved by the network. A prior mathematical question is how the electrical coupling can act on the oscillator to change the times spent in the active and inactive phases. This was the question addressed by me, Abbott, and Soto-Trevino [29].

In its simplest version, the equations we considered have the form of equations from the previous simulation, namely

$$
\begin{aligned}
v^{\prime} & =f(v, w)+d(x-v), \\
w^{\prime} & =\varepsilon h(v, w) \\
x^{\prime} & =g(x)+d(v-x) .
\end{aligned}
$$

With $d=0$, the first two equations describe a relaxation oscillator, qualitatively like a van der Pol oscillator, with $f(v, w)=0$ qualitatively like a cubic. Here $v$ is the voltage of the system and $w$ is a slower "recovery" variable. The voltage $x$ of the bistable cells satisfies an equation in which $g(x)$ is a cubic having three zeros, with the outer two stable fixed points for $x^{\prime}=g(x)$. With $d>0$, the equations form a "fast-slow" system like that of the van der Pol oscillator. As in the latter, it can be shown [30] that solutions converge, as $\varepsilon \rightarrow 0$, to "singular trajectories" made up of solutions to the fast equations

$$
\begin{aligned}
v^{\prime} & =f(v, w)+d(x-v), \\
x^{\prime} & =g(x)+d(v-x)
\end{aligned}
$$

(with $w$ treated as a parameter), and the slow equations

$$
w^{\prime}=\varepsilon h(v, w) .
$$

In the latter, $v$ is a function of $w$ obtained by solving

$$
\begin{aligned}
& 0=f(v, w)+d(x-v), \\
& 0=g(x)+d(v-x)
\end{aligned}
$$

along one of the multiple branches of the latter. For the simple two-dimensional van der Pol equations, the singular solution is easily read off from the nullclines, with the jumps between the fast and slow portions of the trajectories occurring at the local extrema of the cubic-shaped curve $f(v, w)=0$. For the three-dimensional system, however, there is no obvious information in the nullclines about the position of the jump points, and how they depend on parameters. This information is exactly what one needs to figure out the time spent on each of the slow branches (active and inactive states of the AB cell). 
It turns out that this information is in the geometry of the equations, but not in an obvious way. The information comes from the structure in the form of the equation that represents the electrically coupled pair. More specifically, on the slow pieces the trajectories (approximately) satisfy (4). The currents created by the electrical coupling are equal and opposite $(d(x-v)=-d(v-x))$; thus, from $(4)$, the $(v, x)$ coordinates of any point along the slow trajectory must satisfy $f(v, w)=-g(x)$, and the $d$ associated with such a point is the "aspect ratio" $|f /(x-v)|=|g(x-v)|$. By plotting the family of curves $v \rightarrow f(v, w)$ and the curve $x \rightarrow-g(x)$ on the same graph, one can (with effort) read off the points of the slow trajectory, as well as the saddle-node point of the fast equations (3); the latter is associated with the fast jump off that portion of the slow trajectory [29]. The analysis thus tells us what properties of the equations for the two cells affect the dynamic behavior of the network.

In addition to showing how the coupling to the bistable element changes the amount of time the oscillator spends on its active and inactive branches, the analysis reveals surprising facts. For example, one might guess that the larger the coupling strength $d$, the larger the "pinning" effect of the electrical coupling. It turns out [29], however, that the maximal effect can occur for some finite $d$. In some circumstances, the network can act as a switch, leaving the bistable elements in a parameter-determined position regardless of initial conditions. Most importantly, the analysis shows how properties of the bistable cell (e.g., shape of the cubic $x \rightarrow-g(x)$, which models properties of the voltage-gated currents of biophysically modeled cells) can affect whether the two cells will synchronize at all, and what the oscillator trajectory will be. This contrasts strongly with other modeling approaches [31] in which the elements are simple on-off devices, and all properties of the network they constitute then depend only on the strengths of the connections.

The techniques can be used to understand behavior in multicompartment models [32], [33] since the connections among different compartments within a neuron are usually modeled as discrete electrical coupling. The techniques can also be used in networks that combine both electrical and chemical synapses [34].

\section{Time scales in chemical synapses.}

6.1. Slow inhibition and synchronization. Until quite recently, the "folk-wisdom" associated with synchronization was that excitation is good for synchronization and inhibition hurts synchronization. It is easy to find circumstances under which that intuition is correct. The case history in Sec. 3 gives an excellent example. It describes fast excitation for a bursting neuron, using "fast threshold modulation" and a synaptic gating function that saturates at a fixed conductance level for high values of the pre-synaptic voltage. Recall that when the synapse is on, its effect is to change the $v$-nullcline of the postsynaptic cell, raising it (and changing its shape) for an excitatory synapse, and lowering it (also with a change of shape) for an inhibitory one. If the coupling between the two cells is excitatory, whenever one cell fires, it changes the nullcline of the lagging one in a way that allows the latter to fire earlier than it would have without the coupling. It was shown in [22], [23] that the difference in the dynamics of the cells before and after the jump to the activated state leads to stable synchronization. Similarly, if the connections 
are inhibitory, the firing of one leads to a change in nullcline that prevents the other cell from immediately firing, and this can be shown to lead to antiphase bursting of the two cells [35], [36].

The intuition behind that folk-wisdom is based on two implicit assumptions:

1) the onset and offset of the action of the synapses are very fast compared with the intrinsic dynamics of the cells;

2) the cells stay in the active and inactive states for a long time compared with the times used to pass from one state to another.

If either of these assumptions fails, then so can the conclusions about synchronization. The importance of the time scales of synaptic currents for synchronization was discovered in the pioneering work of Rinzel and Wang [36], [37], who were trying to understand how some networks of bursting cells can have coherent oscillations even when there is no excitation in the network. (The network in question was the reticular formation of the thalamus [38].) They noted that inhibition in those networks does not shut off quickly after the cells themselves stop being in their active state. The onset of inhibition also takes a significant amount of time after the cells become active. (See [36] for the equations for the synapse.) Wang and Rinzel showed by simulation that the two together could allow synchrony. (For related work on networks of spiking neurons see [39], [40].)

Recently, D. Terman, A. Bose, and I revisited this system to understand the role of the onset and offset time scales in the inhibitory synapses in determining whether the stable synchronization is possible. For these equations, the relevant mathematical structure was the fast-slow nature of the equations, with the synaptic dynamics occurring on the same time scale as the slow recovery dynamics of the neuron. This makes it possible to use geometrical techniques of fast-slow systems to construct singular solutions such as the synchronous one, and to test for stability [22], [42].

The answers that we uncovered [42] were more complex than we expected. We found that there are different parameter regimes in which wholly different sets of parameters govern stability of the synchronous solution. For some parameter regimes, synchrony is achieved (or undermined) by the interplay of the synaptic decay and the cell recovery; in other regimes, the latter is irrelevant but parameters related to the active period of the cell are relevant. Thus, changing parameters that have nothing to do with time scales could change which time scales are relevant for synchrony. So not only does network behavior change, but what controls the behavior also changes.

We also found various other effects that are hard to see from simulations. One was that the onset time is not relevant to whether the synchronous state is stable, but only to the domain of attraction of the synchronous solution. (A different conclusion is argued in papers on spiking networks [39], [40], [41].) Another, that we believe is relevant to larger network behavior, is that common decaying inhibition, even with decay times considerably larger than any intrinsic time scales in the neurons, can have a synchronizing effect even on cells that are wholly uncoupled. One can also see the circumstances under which behavior other than synchrony is displayed.

In larger networks, there is all the behavior of two-cell networks and also some new features. One new feature is known as "clustering", which can occur in networks that are connected entirely by inhibition or by both excitation and inhibition. It is known 
from simulations of several groups that cells receiving inhibition can break into subsets or clusters, with the clusters out of phase with one another, but synchronized within a cluster [43], [44]. This clustering is aided when there is a fast onset of inhibition, which makes the domain of attraction of the synchronous solution small. A functionally important correlate of clustering is that the population rhythm increases; it is roughly a multiple of the frequency of the synchronous network, where the multiple is the number of clusters.

6.2. Functional reorganization in networks. The last story is closely related to the previous one, and its point is to give the flavor of how some of the effects discussed above can play out in still more complicated networks. The punchline of the work is that changes in biophysical properties of the cells, without changing any synaptic properties, can alter how cells process their synaptic input; this in turn can lead to an effective rewiring of the system. I suspect that this kind of thing goes on all over the nervous system, and is an aspect of how the same hard wiring might be capable of a variety of different functionally important behaviors.

The particular example I have in mind comes from rhythms in the thalamus and cortex during sleep [7]. In an early stage of sleep, the rhythm is called "spindling", which occurs in "pockets" of activity with a frequency of about 7-14 hz within a pocket. In a deeper sleep, the rhythm is slower and more regular, with a frequency of about 1-4 hz. Among the major cellular players in these rhythms are two parts of the thalamus. One type are the cells ( $\mathrm{RE}$ ) of the reticular formation, which consists of cells that provide inhibition. The other consists of collections of thalamocortical cells (TC) that provide excitation to the reticular formation and to the cortex (among other projections). There are also cortical cells, which excite both the TC and RE cells. The inhibition from the RE to TC cells interacts with TC cells via two classes of receptors, so-called GABA $A$ and $\mathrm{GABA}_{B}$. The significance of these two classes for the dynamics is that $\mathrm{GABA}_{A}$-mediated inhibition has a relatively fast onset and is quick to decay, while $\mathrm{GABA}_{B}$-mediated inhibition takes significantly longer to turn on and to decay. (See [45] for a schematic of the network.)

In addition to changes in frequency, the dynamic behavior of the cellular players is very different in the two rhythms [7]. During spindling, the TC cells (the ones receiving the inhibition) fire every few cycles of the population rhythm, while during the delta rhythm, they fire with every cycle of the population rhythm. The RE cells fire with the population rhythm during spindling, but very infrequently-only when there is a burst of excitation from the cortex-during the delta rhythm. For the TC cells, spindling corresponds to clustering and the delta rhythm corresponds to population synchrony.

The question that Terman, Bose, and I addressed in [45] was: what might account for the switch? We know that fast-onset inhibition fosters the clustering and can prevent synchrony. Anything that could effectively remove the fast-onset inhibition could create such a change. The mechanism we suggested is indirect, but accounts for all the changes in all the players, as well as the change in the network behavior. Starting from spindling (the clustered state), the proposed sequence of events starts with some change that raises the threshold of excitation needed to get the RE cells to fire. When they stop firing, the fast-acting inhibition then decays away relatively quickly, leaving only the slow-decaying inhibition, which is synchronizing for the TC cells. This amounts to an effective rewiring, 
since the RE cells no longer respond to the TC cells, and therefore no longer give cycle by cycle fast inhibitory input, which created the clustering. The infrequent (less than $1 \mathrm{hz}$ ) but very large input from the cortex acts to periodically excite the RE cells, refreshing the "good" inhibition to permit the synchronized activity to continue. For our purposes here, the point is that a knowledge of effects of time scales or synapses in networks allows one to uncover complicated mechanisms. For related modeling work, see e.g. [46], [47].

7. Summary and further directions. The case studies I presented displayed some of the biological structure that could be captured in mathematical structure; the latter could be used to deduce consequences not at all obvious from the biology.

The first story showed that even small amounts of structure could be important: oscillating cells with rapid transitions between two kinds of states have different properties than cells with sinusoidal wave-forms when hooked up in arrays. For the electrical coupling story, the useable structure was that, away from the fast jumps, the cells coupled have equal and opposite intrinsic currents. For the slow inhibition story, the fast-slow structure of the equations is used to see how the slow synapses modify the qualitative behavior of the network. The second half of that story showed that changes in intrinsic (non-synaptic) parameters of the cells can lead to a "functional reorganization" in which certain connections are effectively removed, and the inhibition in the system has very different effects.

These case studies all deal with fairly simple versions of mathematical neurons and synapses. In addition, there is an enormous variety of other biological structure not incorporated in those equations. These include the roles of special ionic currents associated with rebounds and delays [13], interaction of networks that have different characteristic time scales [48], effects of noise [49], and effects of the more complex dynamics in the synapses [50].

This leads back to a question asked near the beginning of this essay: why is the nervous system so complex? I think it is fair to say that no one yet has a good answer. However, dynamical systems offer both a method (adding to the arsenal of biological methods) and a large challenge: to look for the mathematical structure associated with the biological structure, and then use this to identify the features of the biology that are responsible for a particular behavior.

\section{REFERENCES}

[1] V. Murthy and E. Fetz, Coherent 25- to $35-\mathrm{Hz}$ oscillations in the sensorymotor cortex of awake behaving monkeys, Proc. Nat. Acad. Sci. USA 5690-5674

[2] C. M. Gray, P. Konig, A. K. Engel, and W. Singer, Oscillatory responses in cat visual cortex exhibit inter-columnar synchronization which reflects global stimulus properties, Nature 338, 334337 (1989)

[3] G. Laurent and H. Davidowitz, Encoding of olfactory information with oscillating neural assemblies, Science 265, 1872-1875 (1994)

[4] E. Basar, C. Basar-Eroglu, R. Parnefjord, E. Rahn, and M. Schurmann, Evoked potentials: ensembles of brain induced rhythmicities in the alpha, theta and gamma ranges, in Induced Rhythms in the Brain, E. Basar and T. H. Bullock, eds., Birkhäuser, Boston, 1992

[5] J. O'Keefe, Hippocampus, theta and spatial memory, Current Opinion in Neurobiology 3, 917-924 (1993) 
[6] F. A. Lenz, J. Vitek, and M. DeLong, Role of the thalamus in parkinsonian tremor: evidence from studies in patients and primate models, Stereotactic and Functional Neurosurgery 60, 94-103 (1993)

[7] M. Steriade, D. Contreras, and F. Amzica, Synchronized sleep oscillations and their paroxysmal developments, Trends in the Neurosciences 17, 199-208 (1994)

[8] N. Kopell, N. Ermentrout, and G. B. Ermentrout, Symmetry and phaselocking in chains of weakly coupled oscillators, Comm. Pure Appl. Math. 39, 623-660 (1986)

[9] N. Kopell and G. B. Ermentrout, Phase transitions and other phenomena in chains of oscillators, SIAM J. Appl. Math. 50, 1014-1052 (1990)

[10] Kandel, Schwartz, and Jessel, Principles of Neuroscience, Simon and Schuster, N.Y., 1991

[11] J. Rinzel and G. B. Ermentrout, Analysis of neural excitability and oscillations, in Methods of Neural Modeling, C. Koch and I. Segev, eds., MIT Press, Cambridge, MA, 1989, pp. 135-170

[12] H. Tuckwell, Stochastic Processes in the Neurosciences, SIAM, Philadelphia, 1989

[13] W. Yamada, C. Koch, and P. Adams, Multiple channels and calcium dynamics in Methods of Neuronal Modeling, C. Koch and I. Segev, eds., MIT Press, 1989

[14] W. Rall, Cable theory for dendritic neurons, in Methods of Neuronal Modeling, C. Koch and I. Segev, eds., MIT Press, 1989

[15] D. A. McCormick, Neurotransmitter actions in the thalamus and cerebral cortex and their role in the neuromodulation of thalamocortical activity, Prog. Neurobiol. 39, 337-388 (1992)

[16] R. Harris-Warrick and E. Marder, Modulation of neural networks for behavior, Ann. Rev. Neurosci. 14, 39-57 (1991)

[17] G. LeMasson, E. Marder, and L. F. Abbott, Activity-dependent regulation of conductances in model neurons, Science 259, 1915-1917 (1993)

[18] C. Morris and H. Lecar, Voltage oscillations in the barnicle giant muscle fiber, Biophysical J. 35, 193-213 (1981)

[19] J. Rinzel, Excitation dynamics: insights from simplified network models, Federation Proc. 44, 2944-2946 (1985)

[20] F. Hoppensteadt and E. Izhikevich, Weakly Connected Neural Networks, Springer-Verlag, N.Y., 1997

[21] G. B. Ermentrout and N. Kopell, Multiple pulse interactions and averaging in coupled neural oscillators, Journal of Math. Biol. 29, 195-217 (1991)

[22] D. Somers and N. Kopell, Rapid synchronization through fast threshold modulation, Biol. Cybern. 68, 393-407 (1993)

[23] D. Somers and N. Kopell, Waves and synchrony in arrays of oscillators of relaxation and nonrelaxation type, Physica D 89, 169-183 (1995)

[24] D. Terman and D. L. Wang, Global competition and local cooperation in a network of neural oscillators, Physica D 81, 148-176 (1995)

[25] C. M. Gray, Synchronous oscillations in neuronal systems: mechanisms and functions, J. Comput. Neuro. 1, 11-38 (1994)

[26] D. L. Wang and D. Terman, Image segmentation based on oscillatory correlation, Neural Comp. 9, 805-836 (1997)

[27] R. Harris-Warrick, E. Marder, A. Selverston, and M. Moulins, eds., Dynamic Biological Networks: The Stomatogastric Nervous System, MIT Press, 1992

[28] L. Abbott, E. Marder, and S. Hooper, Oscillating networks: control of burst duration by electrically coupled neurons, Neural Comp. 3, 487-497 (1991)

[29] N. Kopell, L. Abbott, and C. Soto-Trevino, On the behavior of a neural oscillator electrically coupled to a bistable cell, Physica D, to appear

[30] E. F. Mischenko and N. Kh. Rozov, Differential Equations with Small Parameters and Relaxation Oscillations, Plenum Press, N.Y., 1980

[31] J. J. Hopfield, Neural networks and physical systems with emergent collective computational abilities, Proc. Nat. Acad. Sci. USA 79, 2554-2558 (1982)

[32] V. Booth and J. Rinzel, A minimal compartmental model for a dendritic origin of bistability of motoneuron firing patterns, J. Comput. Neurosci. 2, 1-14 (1995)

[33] I. Segev, J. Fleshman, and R. Burke, Compartmental models of complex neurons, in Methods of Neuronal Modeling, C. Koch and I. Segev, eds., MIT Press, 1989

[34] C. Soto-Trevino, Geometric methods for periodic orbits in singularly perturbed systems, Thesis, Boston University, 1997 
[35] W. Friesen, Reciprocal inhibition, a mechanism underlying oscillatory animal movements, Neurosci. Behavior 18, 547-553 (1994); P. Rowat and A. Selverston, Oscillatory mechanisms in pairs of neurons connected with fast inhibitory synapses, J. Comput. Neurosci. 4, 103-127 (1997); F. Skinner, N. Kopell, and E. Marder, Mechanisms for oscillations and frequency control in networks of mutually inhibitory relaxation oscillators, J. Comput. Neurosci. 1, 69-87 (1994)

[36] X. J. Wang and J. Rinzel, Alternating and synchronous rhythms in reciprocally inhibitory model neurons, Neural Comp. 4, 84-97 (1992)

[37] X. J. Wang and J. Rinzel, Spindle rhythmicity in the reticularis thalami nucleus: synchronization among mutually inhibitory neurons, Neuroscience 53, 899-904 (1993)

[38] M. Steriade, E. G. Jones, and R. R. Llinas, Thalamic oscillations and signaling, Wiley, New York, 1990

[39] C. van Vreeswijk, L. Abbott, and G. B. Ermentrout, When inhibition, not excitation synchronized neural firing, J. Comput. Neurosci. 1, 313-321 (1994)

[40] W. Gerstner, J. L. van Hemmen, and J. Cowen, What matters in neuronal locking?, Neural Comp. 8, 1653-1676 (1996)

[41] C. Chow, Phase-locking in weakly heterogeneous neuronal networks, Physica D 118, 343-370 (1998)

[42] D. Terman, N. Kopell, and A. Bose, Dynamics of two mutually coupled slow inhibitory neurons, Physica D, to appear

[43] D. Golomb and J. Rinzel, Clustering in globally coupled inhibitory neurons, Physica D 72, 259282 (1994); X.-J. Wang, D. Golomb, and J. Rinzel, Emergent spindle oscillations and intermittent burst firing in a thalamic model: specific neuronal mechanisms, Proc. Natl. Acad. Sci. (USA) 92, $5577-5581$ (1995)

[44] N. Kopell and G. LeMasson, Rhythmogenesis, amplitude modulation and multiplexing in a cortical architecture, Proc. Nat. Acad. Sci (USA) 91, 10586-10590 (1994)

[45] D. Terman, A. Bose, and N. Kopell, Functional reorganization in thalamocortical networks: Transition between spindling and delta sleep rhythms, Proc. Nat. Acad. Sci. USA 93, 15417-15422 (1996)

[46] A. Destexhe, D. A. McCormick, and T. J. Sejnowski, A model for 8-10 spindling in interconnected thalamic relay and reticularis neurons, Biophysical J. 65, 2473-2477 (1993)

[47] D. Golomb, X.-J. Wang, and J. Rinzel, Synchronization properties of spindle oscillations in a thalamic reticular nucleus model, J. Neurophys. 72 (1994)

[48] Y. Manor, F. Nadim, E. Marder, S. Epstein, J. Ritt, and N. Kopell, Frequency regulation of a slow rhythm by a fast periodic input: a geometric analysis, submitted

[49] J. Collins, C. Chow, and T. Imhoff, Stochastic resonance without tuning, Nature 376, 236-238 (1995)

[50] L. F. Abbott, J. A. Varela, K. Sen, and S. B. Nelson, Synaptic depression and cortical gain control, Science 275, 220-224 (1997) 